\title{
Partitioning of interaction-induced nonlinear optical properties of molecular complexes. II. Halogen-bonded systems $\dagger$
}

\author{
Miroslav Medved', $\quad$ *ab Alex Iglesias-Reguant, $\quad$ 'Heribert Reis, ${ }^{d}$ \\ Robert W. Góra, e Josep M. Luis ${ }^{\circ}$ and Robert Zaleśny *
}

Following our study on hydrogen-bonded (HB) complexes [Phys. Chem. Chem. Phys., 2018, 20, 19841], the physical nature of interaction-induced (non)linear optical properties of another important class of molecular complexes, namely halogen-bonded (XB) systems, was analyzed in this study. The excess electronic and nuclear relaxation (hyper)polarizabilities of nine representative XB complexes covering a wide range of halogen-bond strengths were computed. The partitioning of the excess properties into individual interaction-energy components (electrostatic, exchange, induction, dispersion) was performed by using the variational-perturbational energy decomposition scheme at the MP2/aug-cc-pVTZ level of theory and further supported by calculations with the SCS-MP2 method. In the case of the electronic interaction-induced properties, the physical composition of $\Delta \alpha^{\text {el }}$ and $\Delta \gamma^{\text {el }}$ was found to be very similar for the two types of bonding, despite the different nature of the binding. For $\Delta \beta^{\mathrm{el}}$, the XB complexes exhibit a more systematic interplay of interaction-energy contributions compared to the HB systems studied in the previous work. Our analysis revealed that the patterns of interaction-energy contributions to the interaction-induced nuclear-relaxation contributions to the linear polarizability and the first hyperpolarizability are very similar. For both properties the exchange repulsion term is canceled out by the electrostatic and delocalization terms. The physical composition of these contributions is analogous to those observed for the HB complexes.

\section{Introduction}

Non-linear optical (NLO) materials based on molecular crystals and/or thin molecular films represent highly attractive alternatives to common ionic inorganic NLO crystals ${ }^{1,2}$ for diverse applications in laser technology, high-speed information transfer and processing, optical data storage, medical diagnostics and

\footnotetext{
${ }^{a}$ Department of Chemistry, Faculty of Natural Sciences, Matej Bel University, Tajovského 40, SK-97400 Banská Bystrica, Slovak Republic. E-mail: miroslav.medved@umb.sk

${ }^{b}$ Regional Centre of Advanced Technologies and Materials, Faculty of Science, Palacký University in Olomouc, Šlechtitelů 27, 78371 Olomouc, Czech Republic

${ }^{c}$ Institute of Computational Chemistry and Catalysis and Department of Chemistry, University of Girona, Campus de Montilivi, 17003 Girona, Catalonia, Spain. E-mail: josepm.luis@udg.edu

${ }^{d}$ Institute of Chemical Biology, National Hellenic Research Foundation (NHRF), Vassileos Constantinou Ave 48th, 11635 Athens, Greece

${ }^{e}$ Department of Physical and Quantum Chemistry, Faculty of Chemistry, Wroclaw University of Science and Technology, Wybrzeże Wyspiańskiego 27, 50-370 Wrocław, Poland.E-mail: robert.zalesny@pwr.edu.pl
}

biological imaging. ${ }^{3-5}$ The possibility of enhancing and tuning the NLO responses of such materials by chemical modifications has fostered the bottom-up design of new molecular systems with extraordinarily large first and/or second hyperpolarizabilities, ${ }^{6-8}$ including push-pull systems, ${ }^{9}$ conjugated asymmetric oligomers, ${ }^{10,11}$ chiral systems, ${ }^{12}$ octupolar chromophores, ${ }^{13}$ and open-shell systems. ${ }^{14}$ However, it has become evident that it is by no means straightforward to derive or even estimate the macroscopic properties of a bulk material from the characteristics of individual molecules, as the NLO properties (NLOPs) of molecular complexes are usually not simply additive. For example, the symmetry and the structure of the crystal are crucial for its NLOPs, especially in the case of the quadratic NLO responses. Another key factor that determines the magnitude of the NLOPs of the bulk material is the effect played by intermolecular interactions, which can substantially change first and second hyperpolarizabilities of a molecular complex compared to those of the isolated subsystems. ${ }^{15-18}$ This change, which often leads to a substantial enhancement of the sum of the properties of the subsystems, may be referred to as the excess property, and is usually analyzed in terms of a few fundamental interaction types based on the 
partitioning of the supermolecular interaction energy into physically meaningful components (e.g., electrostatics, exchange, induction, dispersion). ${ }^{19-26}$ Starting from pioneering works by Fowler and Sadlej, ${ }^{27}$ Bishop and Dupuis ${ }^{28}$ and Heijmen et al., ${ }^{29}$ the excess properties of a wide pallette of systems including hydrogen-bonded dimers ${ }^{30-32}$ and weakly-bound stacked systems ${ }^{33,34}$ were studied, with a focus on the decomposition of the pure electronic component of the properties. In general, it was observed that the electronic excess polarizabilities are governed by the first-order electrostatic term and to a lesser extent by the induction contribution. The effect of these two interaction types is attenuated by the exchange term..$^{30-32}$ On the other hand, no regularities emerged in the case of higher-order excess properties. For example, comparable values of the electronic excess first hyperpolarizability of the urea and diformamide dimers resulted from a very different interplay between interaction-energy contributions. $^{32}$

Another important aspect that needs to be taken into account to obtain a complete picture of NLO responses of molecular systems is the effect of nuclear motions. It has been demonstrated that the magnitude of vibrational contributions to first and second hyperpolarizabilities can be comparable or even prevail over the electronic counterparts provided that static electric fields are involved. ${ }^{35}$ It may be expected that the effect of nuclear motions will be even more pronounced in the case of weakly-bound complexes, as the weak interactions imply the presence of low-frequency vibrational modes which can significantly increase the vibrational NLOPs. Indeed, it was shown in a pilot study on the vibrational contributions to static excess properties of the HCN dimer ${ }^{36}$ that the nuclear-relaxation contribution to the excess first hyperpolarizability is four times larger than the electronic contribution. In addition, it was observed that the composition of excess nuclear-relaxation properties of different order (i.e., dipole polarizability and first and second hyperpolarizability) is very similar. The first-order electrostatic contribution to the excess properties canceled out the exchange repulsion term to a large extent, so that the final values were governed by the induction and dispersion terms. These findings were later confirmed by a more extensive study including ten hydrogen-bonded (HB) systems. ${ }^{37}$ Let us recall that no such regularity has ever been observed in the case of electronic excess higher-order properties.

To extend our understanding of the effect of intermolecular forces on the NLO responses of molecular complexes, here we treat another important type of noncovalent interactions, namely the halogen bond $(\mathrm{XB}),{ }^{38-43}$ which role in material science, structural chemistry, organic synthesis and organocatalysis, polymer science, biochemistry, and medicinal chemistry has been extensively examined in the last decade. ${ }^{43-51}$ This directional non-covalent interaction $(\mathrm{A}-\mathrm{X} \cdots \mathrm{B})$ between a halogen atom (X) acting as a Lewis acid (electron acceptor, or "halogen bond donor") and a Lewis base (electron donor, or "halogen bond acceptor", B) has been successfully interpreted by the concept of the $\sigma$-hole, introduced by Clark et al. in $2007 .{ }^{52}$ The electron density distribution around the halogen atom $\mathrm{X}$, which is covalently bound to an atom (A), is not isotropic, but exhibits a small region of positive electrostatic potential, i.e., a positive domain called the $\sigma$-hole, located on the opposite side of the atom A. This region attracts the lone pair of the Lewis base leading to a potential energy minimum for the A-X $\cdots \mathrm{B}$ angle equal to $180^{\circ}$, imposing the linear arrangement. Let us note that a similar non-covalent bonding pattern was also found for elements of the groups IV, V and VI. ${ }^{53-56}$

There is a strong theoretical and experimental evidence that HB and XB share several common features. ${ }^{56-59}$ In both cases, the $\sigma^{*}$ orbital of an $\mathrm{A}-\mathrm{H}$ or $\mathrm{A}-\mathrm{X}$ donor interacts with a lone-pair orbital of a Lewis base acting as the HB or XB acceptor. Then, depending on the nature of an accompanying atom (group) $\mathrm{A}$ and acceptor $\mathrm{B}$, the $\mathrm{H} \cdots \mathrm{B}$ and $\mathrm{X} \cdots \mathrm{B}$ interactions can change from a weak electrostatic attraction of about $1 \mathrm{kcal} \mathrm{mol}^{-1}$, to a bonding with substantial covalent contribution with binding energies of roughly $40 \mathrm{kcal} \mathrm{mol}^{-1} \cdot{ }^{38-42,55,57}$ However, there are also important differences between $\mathrm{HB}$ and XB. First, there is obviously a larger variety of XBs with different properties, because $\mathrm{X}$ can vary from $\mathrm{F}$, through $\mathrm{Cl}, \mathrm{Br}$, I up to At, differing substantially in their electronic properties (e.g., electronegativity, size, polarizability). In addition, $\mathrm{XB}$ complexes exhibit a more complex interplay between the strength of the $\mathrm{A}-\mathrm{X}$ and $\mathrm{X} \cdots \mathrm{B}$ bonds. Although there exist a few weak HB systems in which A-H bonds become shorter upon the formation of the complex ("blue-shifting HBs"), ${ }^{60,61}$ typically, the $\mathrm{A}-\mathrm{H}$ bond becomes weaker by the formation of the hydrogen bond, resulting in an increased A-H bond length and a red-shift of the frequency of the corresponding stretching vibrational mode. The formation of a halogen bond, however, may lead both to an elongation or a contraction of the A-X bond. ${ }^{62,63}$ This observation was explained by Wang and Hobza ${ }^{64}$ in terms of an interplay between the electron-density transfer to the A-X $\sigma^{*}$ antibonding orbital (the so-called hyperconjugation effect) and electrostatic interactions. If the hyperconjugation effect is dominant, as in the case of strong XBs involving iodine, the A-X bond is elongated. If the hyperconjugation effect is weak, as in many chlorine as well as some bromine containing XB complexes, the electrostatic interaction governs the change of the $\mathrm{A}-\mathrm{X}$ bond length, which is determined by the partial charge on the atom A. A positive partial charge on $\mathrm{A}$ induces a $\mathrm{A}-\mathrm{X}$ bond contraction, whereas a negative charge on $\mathrm{A}$ results in $\mathrm{A}-\mathrm{X}$ elongation. The electrostatic origin of the A-X contraction/elongation is obvious when $\mathrm{X}$ is negatively charged. If the partial charges on the halogen and on A are both positive, the latter charge is usually far larger than the former, and the $\mathrm{A}-\mathrm{X}$ contraction can be explained by the electrostatic attraction between A and B. Thus, XB complexes can be classified as red-shifting or blue-shifting XBs.

In the present study, we have selected nine representative linear $\mathrm{XB}$ complexes belonging to the $\mathrm{XB} 18 \mathrm{set}^{65}$ covering a wide range of $\mathrm{XB}$ binding energies (from $1.41 \mathrm{kcal} \mathrm{mol}^{-1}$ for $\mathrm{HBr} \cdots \mathrm{NCH}$ up to $9.45 \mathrm{kcal} \mathrm{mol}^{-1}$ for $\left.\mathrm{FI} \cdots \mathrm{NCH}\right)$. The dipole moment of the donors in our set may point towards the acceptor $(\mathrm{HBr}, \mathrm{HI})$, away from it (FBr, ClBr, FI, ClI, BrI), or may be zero $\left(\mathrm{Br}_{2}\right.$ and $\left.\mathrm{I}_{2}\right)$. Taking into account the partial charges of $\mathrm{A}$ and $\mathrm{X}$ and the type of $\mathrm{X}$ atom, we may expect that most of the complexes are red-shifting, with the possible exception of $\mathrm{HBr} \cdots \mathrm{NCH}$, which exhibits features which may lead to a blue shift (vide infra). The linearity of the 
selected systems enabled us to apply reliable electron correlation methods for both electronic and nuclear-relaxation longitudinal (hyper)polarizabilities and perform the interaction-induced property decomposition in a well-defined manner. Let us also underline that in all cases the linear arrangement corresponds to the equilibrium geometry of the system.

The article is organized as follows. First, we briefly describe our strategy to compute the excess electronic and nuclear-relaxation properties and their decomposition into interaction-energy components by combining the finite-field nuclear relaxation (FF-NR) method proposed by Bishop, Hasan and Kirtman ${ }^{66,67}$ with the variational-perturbational interaction energy decomposition scheme (VP-EDS). ${ }^{68-71}$ Subsequently, software and computational details are provided. The results section starts with the characterization of the studied systems in terms of the XB strength and the structural features, focusing on the elongation/contraction of the $\mathrm{A}-\mathrm{X}$ bond. Then, the magnitude of the excess electronic and nuclear-relaxation properties of increasing order and the role of individual interaction types in them are thoroughly examined.

\section{Theory}

Our strategy for computing the properties of interest closely follows the procedure described in our previous works on $\mathrm{HB}$ systems. ${ }^{36,37}$ Therefore, here we only provide definitions of quantities discussed later in this study.

The total property $P^{\text {tot }}\left(P^{\text {tot }}=\alpha, \beta, \gamma\right)$ can be split into electronic $P^{\mathrm{el}}$, nuclear-relaxation $P^{\mathrm{nr}}$, and curvature $P^{\text {curv }}$ contributions: ${ }^{72}$

$$
P^{\mathrm{tot}}=P^{\mathrm{el}}+P^{\mathrm{nr}}+P^{\mathrm{curv}}
$$

Whereas $P^{\mathrm{el}}$ describes the pure electronic response to an external electric field at the equilibrium geometry, $P^{\mathrm{nr}}$ and $P^{\text {curv }}$ arise from the change of the electronic and vibrational energies due to the field-induced change of the equilibrium geometry and the shape of the potential energy surface, respectively. The nuclear relaxation contributions to $\alpha, \beta$ and $\gamma$ contain all the lowest-order terms of each type of Bishop-Kirtman brackets contributions, ${ }^{73}$ which include the harmonic corrections for $\alpha$, and the harmonic and lowest-order anharmonic corrections for $\beta$ and $\gamma$. The curvature contributions, which include the rest of the anharmonicity, are computationally far more expensive and usually smaller than $P^{\mathrm{el}}$ and $P^{\mathrm{nr}}$. To compute $P^{\mathrm{nr}}$, we employed the finite-field nuclear-relaxation (FF-NR) method proposed by Bishop, Hasan and Kirtman, ${ }^{67,74}$ which requires optimizing the field-dependent geometry of the molecules maintaining the Eckart conditions ${ }^{75}$ (i.e. not allowing the rotation of the molecule). These field-dependent optimizations were carried out using the procedure developed by Luis et al. ${ }^{76}$

The interaction-induced (excess) property $\Delta P$ (where $P=P^{\text {tot }}$, $\left.P^{\mathrm{el}}, P^{\mathrm{nr}}\right)$ is defined as the difference between the property $P^{\mathrm{AB}}$ of the complex and the sum of the properties $P^{\mathrm{i}}(\mathrm{i}=\mathrm{A}, \mathrm{B})$ of the isolated subsystems at the geometry of the complex:

$$
\Delta P=P^{\mathrm{AB}}-\left(P^{\mathrm{A}}+P^{\mathrm{B}}\right)
$$

Taking into account that the static electric (hyper)polarizabilitities are defined as derivatives of the energy with respect to the external electric field, both $\Delta P^{\mathrm{el}}$ and $\Delta P^{\mathrm{nr}}$ can be split into contributions due to the various intermolecular-interactions types, as we have shown in our recent study. ${ }^{36}$

In principle, any partitioning scheme used for the decomposition of supermolecular interaction energy ${ }^{19-26}$ can be applied with our approach. In this work, we rely on a variational-perturbational decomposition scheme (VP-EDS), ${ }^{71,77-79}$ in which the interpretation of the individual interactions is based on intermolecular perturbation theory. ${ }^{80}$ In VP-EDS, the total interaction energy obtained in a supermolecular approach is partitioned into a selection of interaction energy terms as defined in symmetry-adapted perturbation theory (SAPT). ${ }^{71,77-79}$ At the second-order Møller-Plesset perturbation theory level (MP2), the total VP-EDS interaction energy of the dimer calculated by a supermolecular approach in the dimer-centered-basis set (DCBS) ${ }^{81}$ is partitioned into the Hartree-Fock (HF) and the electron correlation interaction energy components:

$$
\Delta E_{\mathrm{int}}^{\mathrm{MP} 2}=\Delta E_{\mathrm{int}}^{\mathrm{HF}}+\Delta E_{\mathrm{corr}}^{\mathrm{MP} 2}
$$

The HF term can be further partitioned into the electrostatic interactions of unperturbed monomer charge densities, $\varepsilon_{\mathrm{el}}^{(10)}$, the exchange repulsion $\left(\Delta E_{\mathrm{ex}}^{\mathrm{HL}}\right)$ and the charge delocalization $\left(\Delta E_{\mathrm{del}}^{\mathrm{HF}}\right)$, accounting for the exchange effects due to the Pauli antisymmetry principle and induction, respectively.

$$
\Delta E_{\mathrm{int}}^{\mathrm{HF}}=\varepsilon_{\mathrm{el}}^{(10)}+\Delta E_{\mathrm{ex}}^{\mathrm{HL}}+\Delta E_{\mathrm{del}}^{\mathrm{HF}}
$$

The second-order electron correlation term, $\Delta E_{\mathrm{corr}}^{\mathrm{MP} 2}$ :

$$
\Delta E_{\mathrm{corr}}^{\mathrm{MP} 2}=\varepsilon_{\mathrm{el}, \mathrm{r}}^{(12)}+\varepsilon_{\mathrm{disp}}^{(20)}+\Delta E_{\mathrm{ex}}^{(2)}
$$

includes the second-order dispersion interaction, $\varepsilon_{\text {disp }}^{(20)}$, as well as the electron correlation correction to the first-order electrostatic interaction, $\varepsilon_{\mathrm{el}, \mathrm{r}}^{(12)}$, and the remaining second-order electron correlation effects $\left(\Delta E_{\mathrm{ex}}^{(2)}\right)$. The latter term accounts mainly for the uncorrelated exchange-dispersion and electron correlation corrections to the Hartree-Fock exchange repulsion. ${ }^{79,80}$ The terms $\varepsilon_{\mathrm{el}}^{(10)}$ and $\varepsilon_{\text {disp }}^{(20)}$ are obtained using standard polarization perturbation theory, whereas the term $\varepsilon_{\mathrm{el}, \mathrm{r}}^{(12)}$ is calculated using the formula proposed by Moszyński et al. ${ }^{82}$ The numbers $n, m$ in the superscript of terms such as $\varepsilon_{\mathrm{el}}^{(\mathrm{nm})}$ refer to orders of perturbation in the intermolecular interaction operator $(n)$ and intramonomer correlation operator $(m)$, respectively.

\section{Software and computational details}

Field-free equilibrium structures of all systems were obtained by geometry optimizations with tight convergence criteria $\left(10^{-12}\right.$ Hartree and $10^{-9}$ Hartree Bohr ${ }^{-1}$ for energy and gradients, respectively) and employing the MP2 method in combination with Dunning's correlation-consistent aug-cc-pVTZ basis set. ${ }^{83}$ For the heavy atoms (bromine and iodine), the corresponding effective core potential (aug-cc-pVTZ-PP) ${ }^{84}$ was applied to include scalar relativistic effects. It was shown by Kozuch and Martin, ${ }^{65}$ that relativistic ECP in combination with the non-relativistic Hamiltonian provides binding energies of XB systems in very good agreement with those obtained using the all-electron 
Douglas-Kroll (DK) Hamiltonian. Harmonic vibrational analysis was performed at the same level of theory to confirm that the optimized structures were true minima. All field-free computations were performed with the Gaussian program. ${ }^{85}$ Fielddependent geometry optimizations and property calculations were carried out using custom computer programs enforcing again tight convergence thresholds. ${ }^{86,87} 50$ iterations during the FF-NR procedure were sufficient to achieve the convergence $10^{-13}$ a.u. on energy and $10^{-7}-10^{-10}$ Hartree Bohr ${ }^{-1}$ on RMS gradient for the full mesh of fields. VP-EDS calculations were performed using a modified in-house version of the Gamess (US) program. ${ }^{88,89}$ As described in the previous section, the VP-EDS partitioning of the excess properties is fully consistent with the utilization of the MP2 method for the evaluation of the total electronic and nuclearrelaxation properties of complexes. Our choice of the aug-cc-pVTZ basis set was based on recent studies by some of us, ${ }^{36,90}$ which showed that it provides reliable results for both electronic and nuclear-relaxation (hyper)polarizabilities of small molecules and HB dimers with average errors below 1\% compared to values obtained with the aug-cc-pVQZ basis set. Our choice is also supported by the benchmark study of XB systems by Kozuch and Martin, ${ }^{65}$ which claimed that for practical purposes the aug-ccpVTZ basis set is sufficient. Furthermore, we also confirmed the basis set saturation of our NLOP data by comparing the results obtained with the aug-cc-pVTZ and aug-cc-pVQZ basis sets (corresponding data are shown in ESI, $\uparrow$ Table S1). The relative percentage error in electronic $\alpha, \beta$ and $\gamma$ for the aug-cc-pVTZ basis set is only $0.1 \%,-4.4 \%$ and $-2.8 \%$, respectively. Let us recall that the FF-NR approach requires the application of the same level of theory both for the field-dependent geometry optimization and property calculations, which puts some restrictions on the level of correlation as well as on the size of the basis sets we can apply. However, it is known that MP2 tends to overestimate the binding of weakly bound systems. ${ }^{91}$ Therefore, we also applied the spincomponent-scaled variant of MP2 (SCS-MP2), ${ }^{92}$ which scales down the same spin and enlarges the opposite spin components of the MP2 correction, to calculate the electronic and nuclear-relaxation properties of three representative systems of our set (see Table S2 in ESI $\dagger$ ). The SCS-MP2 method was shown to provide bond lengths and dissociation energies of XB complexes in excellent agreement with results obtained with the $\operatorname{CCSD}(\mathrm{T})$ method. ${ }^{65}$ Our comparison of MP2 with SCS-MP2 results revealed that the former only slightly overestimates both electronic and nuclear-relaxation contributions to (hyper)polarizabilities (by ca. 10-18\%). The only somewhat larger discrepancy was observed for $\beta^{\text {el }}$ of $\mathrm{FBr} \cdots \mathrm{NCH}$, but the magnitude of this property is rather small. To sum up, we can conclude that MP2 is a reliable method for the analysis of both electronic and vibrational properties of XB complexes and, in particular, for unravelling the interplay of interaction types contributing to the excess (hyper)polarizabilities.

\section{Results and discussion}

Before performing a detailed analysis of excess properties, let us briefly discuss the energetics and structural aspects of the

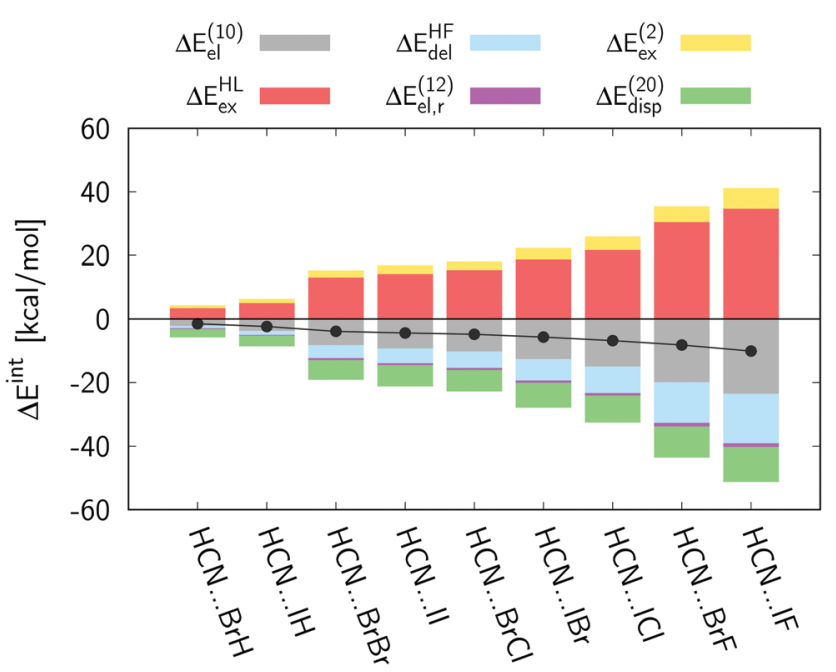

Fig. 1 Interaction energy partitioning for the studied species at the equilibrium geometries optimized using MP2/aug-cc-pVTZ-PP level of theory. The numerical data can be found in ESI. $\dagger$

halogen bonding in the systems studied. Fig. 1 shows the VP-EDS interaction energies (IEs) and their components for the whole set of complexes ordered according to increasing values of the total interaction energy. Let us note that our results agree with the trends reported by Kozuch and Martin for binding energies obtained with the CCSD(T)/CBS method ${ }^{65}$ (see Table S3, ESI $\dagger$ ) thus further supporting the suitability of the MP2 method for interaction-energy decomposition. As expected, the magnitude of the VP-EDS/MP2 interaction energies is in general larger for the iodine-bonded complexes. The interaction energies correlate with the vibrational frequency of the $\mathrm{X} \cdots \mathrm{B}$ stretching mode as well as with the partial charge on the halogen atom of the isolated $\mathrm{XB}$ donor $\left(q_{\mathrm{x}}\right)$ obtained by natural orbital analysis (see Table S4 and panels (a) and (b) in Fig. S1, ESI $\dagger$ ). The set of our complexes can thus be split into three subgroups: (i) weakly-bound systems involving $\mathrm{XB}$ donor with a negative $q_{\mathrm{X}}$ and a positive $q_{\mathrm{A}}(\mathrm{HBr} \cdots \mathrm{NCH}, \mathrm{HI} \cdots \mathrm{NCH})$; (ii) moderate $\mathrm{XB}$ complexes involving nonpolar XB donors $\left(\mathrm{Br}_{2} \cdots \mathrm{NCH}, \mathrm{I}_{2} \cdots \mathrm{NCH}\right)$; and (iii) strongly-bound $\mathrm{XB}$ systems with positive $q_{\mathrm{X}}$ and negative $q_{\mathrm{A}}$, in which the $\mathrm{XB}$ donor strength increases with increasing difference between the electronegativity values of $\mathrm{A}$ and $\mathrm{X}$, i.e., $\mathrm{ClBr}<\mathrm{BrI}<\mathrm{ClI}<\mathrm{FBr}<$ FI. The fact that the total IEs significantly correlate with the $q_{\mathrm{x}}$ values suggests that electrostatics plays an important role in the halogen bonding. This notion is also supported by even stronger correlation of the firstorder electrostatic IE contribution with $q_{\mathrm{x}}$ (Fig. S1, panel (b), $\mathrm{ESI} \dagger$ ). However, it is evident from Fig. 1 that the (stabilizing) electrostatic term is not the dominant one. In fact, the Pauli exchange-repulsion contributions have different sign but similar absolute magnitude than the sum of the electrostatic and charge delocalization terms. As a result of the nearly complete cancellation of these three terms, the total IEs are mainly governed by the electron correlation effects (predominantly by dispersion forces; see Fig. S1 panel (c), ESI $\dagger$ ), in line with previous studies analyzing the role of individual IE terms in halogen bonding. ${ }^{57,93}$ Indeed, the Hartree-Fock method, which neglects electron 
correlation, incorrectly predicts the complexes $\mathrm{HBr} \cdots \mathrm{NCH}$, $\mathrm{Br}_{2} \cdots \mathrm{NCH}$ and $\mathrm{I}_{2} \cdots \mathrm{NCH}$ to be unbound. In this respect, the current set of XB systems qualitatively differs from a previously studied group of $\mathrm{HB}$ complexes, ${ }^{37}$ in which the two first-order perturbation theory terms (i.e., electrostatic and exchange repulsion) canceled each other out to a large extent and the overall stabilization was mainly due to the negative delocalization and dispersion components. The larger role of the exchange-repulsion in the case of XB systems compared to $\mathrm{HB}$ complexes is related to the presence of lone pairs on the halogen atoms, which are close to the lone pair of the $\mathrm{XB}$ acceptor. Concerning the changes of the $\mathrm{A}-\mathrm{X}$ bond lengths upon complex formation, elongation of the bond is observed in all cases, together with a red-shift of the frequency of the corresponding stretching vibrational mode. In general, the lengthening of the A-X bond increases with $q_{\mathrm{X}}$ (and thus also with the total IE), with a more pronounced correlation for the weakly bound brominebonded complexes, where hyperconjugation effects are smaller (Fig. S1 panel (d), ESI $\dagger$ ). Let us note that even the most weakly bound complex, $\mathrm{HBr} \cdots \mathrm{NCH}$, showing a negative $q_{\mathrm{x}}$ and a positive $q_{\mathrm{A}}$ (thus fulfilling prerequisites for blue-shifting $\mathrm{XBs}$ ), still displays a positive albeit small change of $d(\mathrm{~A}-\mathrm{X})$ and a decrease of the corresponding vibrational frequency upon complex formation. Thus, we conclude that all studied XB complexes can be classified as red-shifting XBs.

Excess electronic dipole moments and their decomposition into individual interaction-energy contributions for the $\mathrm{XB}$ complexes studied are shown in Fig. 2. Let us recall that, by definition, the nuclear-relaxation contribution to this property vanishes. For all the complexes studied the net electronic contribution enhances the absolute magnitude of the excess dipole moment (for the chosen molecular orientation the dipole moment of all the complexes is negative; see Table S5, ESI $\dagger$ ). The magnitude of the total excess values is rather small (lower than 0.3 a.u.) for the two complexes containing hydrogen halides ( $\mathrm{HI}$ and $\mathrm{HBr}$ ), and a bit larger for the other complexes (from 0.64 to 0.94 a.u.), with the largest values pertaining to the iodine-bonded complexes. Clearly, the excess property is

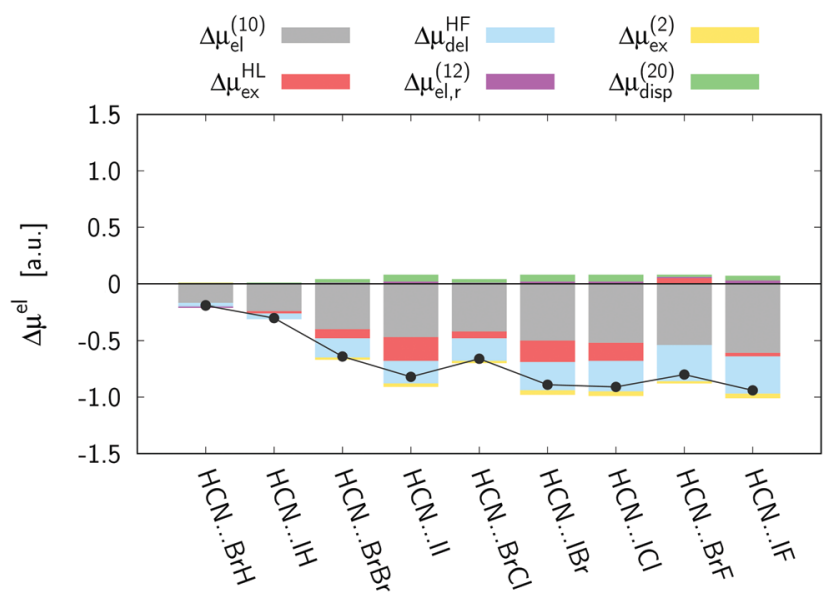

Fig. 2 Breakdown of interaction-induced electronic dipole moment into various interaction types. The numerical data can be found in ESI. $\dagger$ dominated by the first-order electrostatic component. However, although their contributions are rather system-dependent (see also Table S6, ESI $\dagger$ ), the exchange-repulsion and delocalization terms play a more important role, as compared to HB systems. ${ }^{37}$ For all the complexes except $\mathrm{HBr} \cdots \mathrm{NCH}$ and $\mathrm{HI} \cdots \mathrm{NCH}$, the contribution of the latter to the total excess value is larger than $24 \%$. The role of the exchange repulsion is smaller, although when the $\mathrm{X}$ atom is iodine, and the $\mathrm{A}$ atom is $\mathrm{I}, \mathrm{Br}$ or $\mathrm{Cl}$, the exchange contribution is still around $20 \%$. In addition, in the case of the $\mathrm{XB}$ systems the Pauli repulsion contribution has the same sign as the electrostatic component (except for $\mathrm{FBr} \cdots \mathrm{NCH}$, where it is practically negligible), whereas in $\mathrm{HB}$ complexes it has usually the opposite sign. The electron correlation effects are in both types of complexes relatively small.

To explain the observed trends, it is worth noting that the dipole moment of a complex is the result of a rather complicated interplay of the mutual orientation of the dipole moment vectors of the constituting subsystems, and it may also be strongly affected by the mutual polarization (depending mainly on the magnitude of the electronic polarizabilities of the constituents) and possible charge transfer between the subsystems. The small values of the excess dipole moment of $\mathrm{HBr} \cdots \mathrm{NCH}$ and $\mathrm{HI} \cdots \mathrm{NCH}$ result from the anti-parallel directions of the molecular dipole moment vectors, as well as from the fact that their $\mathrm{XB}$ is very weak, which leads to negligible exchange and delocalization contributions. In the case of non-polar XB donors, the increase of the excess dipole moment is mainly due to the polarizability of the halogen-bond donors, which is larger for the iodine molecule. The negative charge localized on the nitrogen atom polarizes the $\mathrm{X}_{2}$ molecule inducing a positive charge in the neighbouring halogen atom and a negative charge on the more distant halogen atom. The Pauli repulsion enhances this polarization (to a larger extent in the case of $\mathrm{I}_{2} \cdots \mathrm{NCH}$ due to a stronger $\mathrm{XB}$ ), so the exchange term increases the magnitude of the excess property. In the remaining complexes with the parallel dipole moment vectors of donor and acceptor molecules, the electrostatic contribution is again related mainly to the polarizabilities of the constituents, which do not significantly differ from those corresponding to the XB complexes with nonpolar donors. The exchange-repulsion contribution enhances the polarization of the $\mathrm{XB}$ donor, thus increasing the total excess property especially in complexes containing iodine atoms. Finally, the delocalization contribution increases with increasing interaction energy, in line with the hypothesis of an increasing role of the hyperconjugation effect along the series.

A comparison of the electronic and nuclear-relaxation dipole polarizabilities shows that the electronic contributions to the total property notably prevail over those related to the nuclear motion (Table S7, ESI $\dagger$ ). The ratios of the $\Delta \alpha^{\mathrm{el}}$ and $\alpha^{\mathrm{el}}$ values indicate that the intermolecular interactions bring about a $10-25 \%$ increase of the property. The pattern of individual contributions to $\Delta \alpha^{\mathrm{el}}$ is very similar to that observed in the case of $\mathrm{HB}$ systems. ${ }^{37}$ In particular, the electrostatic contribution plays a dominant role, whereas the exchange and delocalization terms are smaller in magnitude and have opposite signs. As the correlation effects are only minor, the total electronic excess 
property closely follows the pattern of the electrostatic component. Relative magnitudes of $\Delta \alpha^{\mathrm{el}}$ are similar to $\Delta \mu^{\mathrm{el}}$, i.e., they are notably smaller for the weakly bound and least polarizable HX $\cdots \mathrm{NCH}$ complexes. We note that $\Delta \alpha^{\mathrm{el}}$ values for the remaining systems fluctuate around 20 a.u., with systematically slightly larger values for the iodine-bonded systems.

In the case of strongly bound $\mathrm{XB}$ complexes, the nuclearrelaxation contributions amount to $c a \cdot 20-30 \%$ of the total property of the complex, and the $\Delta \alpha^{\mathrm{nr}}$ values are even larger than the corresponding $\Delta \alpha^{\mathrm{el}}$. The HX $\cdots \mathrm{NCH}$ systems again differ qualitatively from the other complexes by much smaller vibrational effects on the polarizability; the nuclear-relaxation contributions to the total property of the complex are below $10 \%$ (see Table S7, ESI $\dagger$ ), and they are 3-4 times smaller than their electronic counterparts in terms of the excess properties. The partitioning of $\Delta \alpha^{\mathrm{nr}}$ into interaction energy components displayed in Fig. 3 (lower panel) reveals important differences between the physical composition of $\Delta \alpha^{\mathrm{nr}}$ and $\Delta \alpha^{\mathrm{el}}$. Firstly, although the values of both properties are similar in magnitude, the former results from a gentle balance between interaction-energy contributions one order of magnitude larger than for the electronic property. Secondly, the relative weight due to the different intermolecular interaction types are significantly altered. In particular, the electrostatic contribution to $\Delta \alpha^{\mathrm{nr}}$ is far smaller than the corresponding exchange-repulsion term for all $\mathrm{XB}$ complexes investigated. As the two terms have opposite signs, the resulting first-order contribution is negative, and the total $\Delta \alpha^{\mathrm{nr}}$ becomes positive owing to the relatively large positive delocalization contribution and a non-negligible

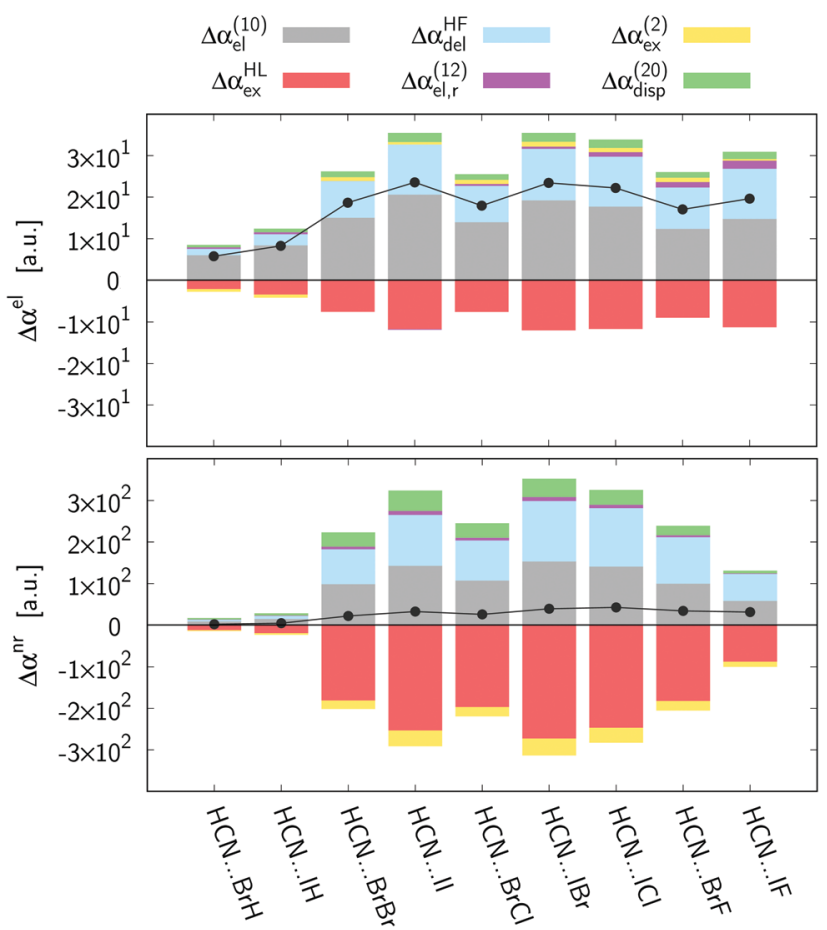

Fig. 3 Breakdown of electronic and nuclear-relaxation contributions to interaction-induced polarizability into interaction types. The numerical data can be found in ESI. $\dagger$ dispersion term. Interestingly, the $\Delta \alpha^{\mathrm{nr}}$ pattern is again very similar to that observed in the case of HB systems despite the differences in the nature of IEs, and the $\Delta \mu^{\mathrm{el}}$ term discussed above: the intermolecular interactions increase the polarizability of the complex with respect to isolated molecules due to both electronic and nuclear-relaxation effects.

The static electronic and nuclear-relaxation first hyperpolarizabilities of the studied XB complexes are reported in Table S8 (ESI $\dagger$ ). Contrary to the case of the polarizabilities, the nuclear relaxation contribution plays a major role here, amounting to $90-95 \%$ of the total property of the complex. Both electronic and vibrational first hyperpolarizabilities are significantly affected by intermolecular interactions and both increase the net hyperpolarizability of all the studied complexes. The excess contributions have in all cases a negative sign (except for $\Delta \beta^{\text {el }}$ of IF $\cdots \mathrm{NCH}$ ), and in general their magnitude more than outweighs the sum of the values of the isolated subsystems, which is either positive or of a small negative value. Thus, the $\beta^{\mathrm{el}}$ and $\beta^{\text {nr }}$ values for nearly all the complexes are negative (again, except for $\beta^{\mathrm{el}}$ of IF $\cdots \mathrm{NCH}$ ). The upper panel of Fig. 4 shows that the magnitude of $\Delta \beta^{\text {el }}$ (the smaller contribution to $\Delta \beta$ ), which does not follow the increasing bond strength of XBs. $\Delta \beta^{\text {el }}$ is rather small for both weakly- as well as strongly-bound $\mathrm{XB}$ systems, and exhibits an enhancement for moderately strong complexes. The largest value for $\Delta \beta^{\mathrm{el}}$ is found for the $\mathrm{I}_{2} \cdots \mathrm{NCH}$ complex. In the case of the two weakly bound complexes, the small values of $\Delta \beta^{\mathrm{el}}$ may be expected since the replacement of a halogen atom by a hydrogen atom leads to a considerably smaller electronic density. However, the explanation of the

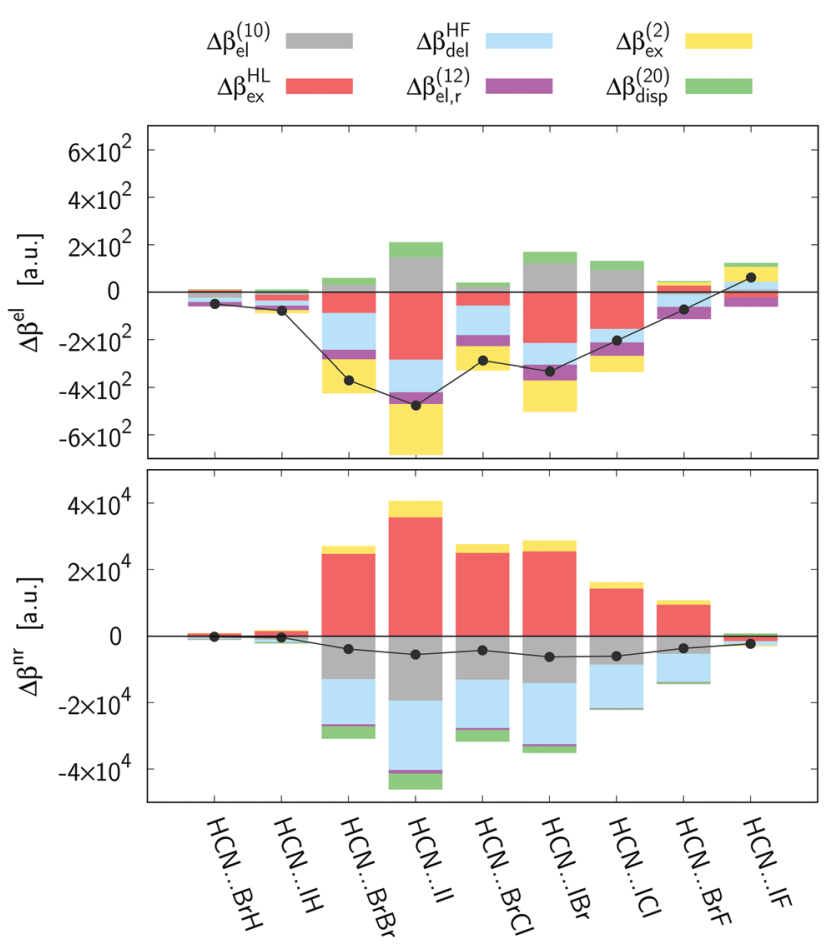

Fig. 4 Breakdown of electronic and nuclear-relaxation contributions to interaction-induced first hyperpolarizability into interaction types. The numerical data can be found in ESI. $\dagger$ 
systematic decrease of excess hyperpolarizability for the most strongly bound complexes is not obvious, as all the interactioninduced components are in this case counter-intuitively small despite the strong interaction. One possible explanation would be that these species are more tightly bound and thus more rigid, but this does not explain why the purely electronic contributions are vanishing. In any case this is quite an interesting observation, indicating that in order to enhance nonlinear response of a molecular crystal the moderately strong interactions are preferred. For the moderately-bound XB systems, some regularities can be observed. Although no dominant component can be identified, electrostatic and dispersion contributions are always positive, whereas the other components are negative. Interestingly, the second-order exchange-correlation term $\left(\Delta \beta_{\mathrm{ex}}^{(2)}\right)$ plays a significant role, and also the electron correlation correction to the first-order electrostatic contribution $\left(\Delta \beta_{\mathrm{el}, \mathrm{r}}^{(12)}\right)$ is not negligible.

The trend of the $\Delta \beta^{\mathrm{nr}}$ values with increasing interaction energy qualitatively follows the corresponding trend observed for $\Delta \beta^{\text {el }}$, i.e., although being in general much larger in magnitude, the two properties are smallest for the complexes characterized by the weakest interactions, increase for moderately-bound systems, and finally drop again for the strongest ones. The partitioning pattern of $\Delta \beta^{\mathrm{nr}}$ is very similar to that observed for $\Delta \alpha^{\mathrm{nr}}$. Again, the dominating exchange-repulsion contribution is slightly overcompensated by the sum of the electrostatic and delocalization components, whereas the electron correlation terms cancel each other out to a large extent. It is also worth noting that the $\Delta \beta^{\text {nr }}$ values result from a gentle balance of relatively large contributions, just as in the case of $\Delta \alpha^{\mathrm{nr}}$.

The evolution of the electronic excess second hyperpolarizability with increasing XB strength shown in Fig. 5 resembles that of the electronic excess polarizability, i.e., the two weakest $\mathrm{XB}$ complexes exhibit rather small values, whereas for the stronger complexes $\Delta \gamma^{\mathrm{el}}$ increases to values ranging from $14.5 \times 10^{3}$ to $45.0 \times 10^{3}$ a.u. Again, the largest value is obtained for the $\mathrm{I}_{2} \cdots \mathrm{NCH}$ complex. The excess property typically

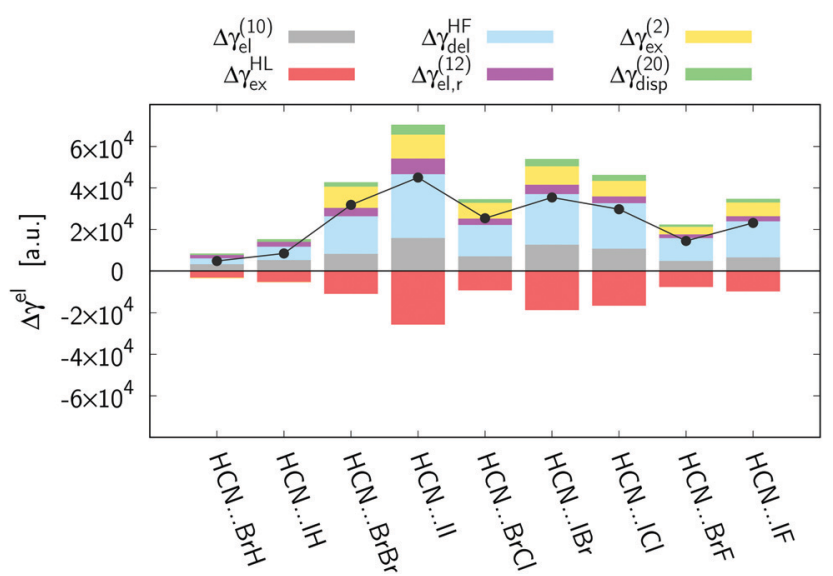

Fig. 5 Breakdown of electronic contribution to interaction-induced second hyperpolarizability into interaction types. The numerical data can be found in ESI. $\dagger$ amounts to $50 \%$ and $70-80 \%$ of the total electronic second hyperpolarizability for the weakly and the strongly bound $\mathrm{XB}$ complexes, respectively. The partitioning of $\Delta \gamma^{\mathrm{el}}$ reveals a rather regular pattern of interaction-energy contributions. The exchangerepulsion, which is of opposite sign than all other components, prevails over the electrostatic term, thus rendering the total contribution of first-order perturbation-theory negative. However, the positive delocalization term dominates over the other components (contributing by $c a .60 \%$ to $\Delta \gamma^{\mathrm{el}}$ ) and brings about a significant increase of the property. As the electron-correlation effects enhance the property further, $\Delta \gamma^{\text {el }}$ attains large positive values. Due to the approximate cancellation between the negative repulsion exchange and the positive electron-correlation correction terms, the sum of the first-order electrostatic and delocalization terms provides a quite reliable rough estimate of the total values of $\Delta \gamma^{\text {el }}$. Due to considerable numerical uncertainties obtained for $\gamma^{\text {nr }}$ and $\Delta \gamma^{\text {nr }}$ (see Table S8, ESI $\dagger$ ), we have not performed the VP-EDS decomposition of the corresponding excess property. Nevertheless, based on relatively stable data for $\mathrm{ClBr} \cdots \mathrm{NCH}$ and $\mathrm{HI} \cdots \mathrm{NCH}$, we can presume that the nuclearrelaxation contributions constitute a major part of the static total second hyperpolarizability of XB complexes.

The results discussed above concern the decomposition of electronic and pseudo nuclear-relaxation contributions to (hyper)polarizabilities. However, according to eqn (1) there are also higher-order anharmonic contributions. Although a method for the decomposition of these contributions has not been developed yet, it is interesting to estimate their magnitude for $\alpha, \beta$ and $\gamma$ of the complexes considered here. The computed values are included in Table S14 in the ESI. $\dagger$ They show that $\alpha^{\text {curv }}$ is for all the complexes far smaller than the corresponding nuclear relaxation contribution $\alpha^{\mathrm{nr}}$. This also holds for $\beta^{\text {curv }}$ and $\gamma^{\text {curv }}$ for the majority of the complexes, with just four exceptions ( $\beta^{\text {curv }}$ and $\gamma^{\text {curv }}$ of $\mathrm{HBr} \cdots \mathrm{NCH}$ and $\gamma^{\text {curv }}$ of $\mathrm{HI} \cdots \mathrm{NCH}$ and $\left.\mathrm{FI} \cdots \mathrm{NCH}\right)$.

\section{Conclusions}

The detailed composition of the excess electronic and nuclearrelaxation (hyper)polarizabilities of a few selected linear XB systems was analyzed at the MP2/aug-cc-pVTZ level of theory by applying the FF-NR method in combination with the VP-EDS approach to evaluate individual contributions due to fundamental interaction types. Our test set comprised weakly bound, moderately bound (which involved nonpolar XB donors) and also strongly bound XB systems, in which the XB strength is enhanced by the dipolar interactions between the donor and the acceptor. The structural and vibrational analyses revealed that all of the systems can be classified as red-shifting XB complexes. In general, the intermolecular interactions consequently enhance the excess electrooptic properties of the studied complexes with respect to isolated species. However, the origins of this enhancement may vary between different systems and for different properties. The excess dipole moment shows a slowly rising tendency with the increasing magnitude of interaction energy, with slightly larger magnitudes for the iodine-bonded complexes. 
As in the HB systems studied in ref. 37, the property is dominated by the electrostatic component, but the exchangerepulsion and delocalization terms are a bit larger in $\mathrm{XB}$ systems. Moreover, the Pauli repulsion term has almost always the same sign as the electrostatic and delocalization components, whereas in the case of $\mathrm{HB}$ complexes it usually acts in the opposite direction.

The (hyper)polarizabilities of the $\mathrm{XB}$ complexes are also significantly affected by the intermolecular interactions. In the case of $\Delta \alpha^{\mathrm{el}}$ and $\Delta \alpha^{\mathrm{nr}}$, they typically amount to $18 \%$ and $99 \%$ of $\alpha^{\text {el }}$ and $\alpha^{\text {nr }}$ of the complex, respectively. The partitioning of $\Delta \alpha^{\mathrm{el}}$ and $\Delta \alpha^{\mathrm{nr}}$ revealed that the former closely follows the pattern of the electrostatic component, whereas the latter results from a gentle balance of order-of-magnitude larger interaction-energy contributions. Similar observations were reported by some of the present authors for HB systems. ${ }^{37}$ In the case of the first hyperpolarizability, the roles of electronic and nuclear-relaxation contributions are dramatically different. Here the latter is far larger than the former and amounts to ca. $90-95 \%$ of the total value of the static property. Moreover, by and large the whole second-order NLO response of these complexes is due to intermolecular forces, implying the importance of the analysis performed to understand the origins of this effect. The $\Delta \beta^{\text {nr }}$ values are small for the weak complexes, increase for moderately-bound systems, and drop again for the most strongly bound ones. Systematically, the Pauli exchangerepulsion term dominates, but is overcompensated by the electrostatic and delocalization components, which have the opposite sign. The electron correlation terms of $\Delta \beta^{\mathrm{nr}}$ cancel out each other to a large extent. The physical nature of $\Delta \beta^{\mathrm{nr}}$ is thus very similar to that observed for $\Delta \alpha^{\mathrm{nr}}$. It is interesting to note that in the case of the first hyperpolarizabilities the excess properties are not only much larger in magnitude than the net property of isolated systems but often change qualitatively the response of the complex to the external electric field (by changing the sign of the resulting first hyperpolarizability of the complex). The relative magnitudes of the excess electronic second hyperpolarizabilities along the series of the studied XB systems are similar to that of $\Delta \alpha^{\text {el }}$. However, the relative electrostatic contribution is notably smaller, which was also observed in the case of HBs.

To sum up, the patterns of interaction-energy contributions to the nuclear-relaxation excess properties of different order are preserved through both sets of $\mathrm{HB}$ and $\mathrm{XB}$ complexes, which is a very interesting and rather surprising finding if one takes into account the completely different nature of the intermolecular interaction of these two types of systems. In the case of the electronic properties, the physical origins of $\Delta \alpha^{\mathrm{el}}$ as well as those of $\Delta \gamma^{\text {el }}$ were also found to be similar for the two groups. For $\Delta \beta^{\mathrm{el}}$, the $\mathrm{XB}$ complexes exhibit more systematic behavior compared to the HB systems.

\section{Conflicts of interest}

The authors declare no competing financial interest.

\section{Acknowledgements}

M. M. acknowledges the Slovak Research and Development Agency (project no. APVV-15-0105). R. Z. and R. W. G. gratefully acknowledge the support from the National Science Centre, Poland (Grant No. 2015/19/B/ST4/01881). J. M. L. is grateful for financial support from the Spanish MICIN PGC2018-098212-B-C22 and the Catalan DIUE 2017SGR39. This research used computational resources of the Wroclaw Center for Networking and Supercomputing and the HPCC of Matej Bel University in Banska Bystrica by using the infrastructure acquired in projects ITMS 26230120002 and 26210120002 supported by the Research and Development Operational Program funded by the ERDF.

\section{References}

1 D. N. Nikogosyan, Nonlinear Optical Crystals: A Complete Survey, Springer-Science + Business Media, B.V., 2005.

2 R. Ganeev, I. Kulagin, A. Ryasnyanskiy, R. Tugushev and T. Usmanov, Opt. Commun., 2004, 229, 403-412.

3 Nonlinear Optical Properties of Matter: From Molecules to Condensed Phases, ed. M. G. Papadopoulos, J. Leszczynski and A. J. Sadlej, Springer, Dordrecht, 2006.

4 L. R. Dalton, P. A. Sullivan and D. H. Bale, Chem. Rev., 2010, 110, 25-55.

5 L. R. Dalton, P. Gunter, M. Jazbinsek, O. Kwon and Ph. A. Sullivan, Organic Electro-Optics and Photonics: Molecules, Polymers, and Crystals, Cambridge University Press, 2015.

6 T. P. Radhakrishnan, Acc. Chem. Res., 2008, 41, 367-376.

7 P. A. Sullivan and L. R. Dalton, Acc. Chem. Res., 2010, 43, 10-18.

8 M. M. Alam, M. Chattopadhyaya, S. Chakrabarti and K. Ruud, Acc. Chem. Res., 2014, 47, 1604-1612.

9 B. L. Davydov, L. D. Derkacheva, V. V. Dunina, M. E. Zhabotinskii, V. F. Zolin, L. G. Koreneva and M. A. Samokhina, JETP Lett., 1970, 12, 16.

10 B. Champagne, D. Jacquemin, J. M. Andre and B. Kirtman, J. Phys. Chem. A, 1997, 101, 3158-3165.

11 D. Jacquemin, M. Medved and E. Perpete, Int. J. Quantum Chem., 2005, 103, 226-234.

12 P. Fischer and B. Champagne, Challenges and Advances in Computational Chemistry and Physics, in Non-linear optical properties of matter: From molecules to condensed phases, 2006, vol. 1, pp. 359-381.

13 B. J. Coe, S. P. Foxon, M. Helliwell, D. Rusanova, B. S. Brunschwig, K. Clays, G. Depotter, M. Nyk, M. Samoc, D. Wawrzynczyk, J. Garín and J. Orduna, Chem. - Eur. J., 2013, 19, 6613-6629.

14 M. Nakano and B. Champagne, Wiley Interdiscip. Rev.: Comput. Mol. Sci., 2016, 6, 198-210.

15 S. D. Bella, M. A. Ratner and T. J. Marks, J. Am. Chem. Soc., 1992, 114, 5842-5849.

16 B. Champagne and D. M. Bishop, Advances in Chemical Physics, John Wiley \& Sons, Inc., 2003, vol. 126, pp. 41-92.

17 A. Facchetti, E. Annoni, L. Beverina, M. Morone, P. Zhu, T. J. Marks and G. A. Pagani, Nat. Mater., 2004, 3, 910-917. 
18 A. Datta and S. K. Pati, Chem. Soc. Rev., 2006, 35, 1305-1323. 19 K. Morokuma, J. Chem. Phys., 1971, 55, 1236-1244.

20 T. Ziegler and A. Rauk, Theor. Chim. Acta, 1977, 46, 1-10.

21 K. Kitaura and K. Morokuma, Int. J. Quantum Chem., 1976, 10, 325-340.

22 P. S. Bagus, K. Hermann and C. W. Bauschlicher, J. Chem. Phys., 1984, 80, 4378.

23 W. J. Stevens and W. H. Fink, Chem. Phys. Lett., 1987, 139, 15-22.

24 E. D. Glendening and A. Streitwieser, J. Chem. Phys., 1994, 100, 2900.

25 Y. Mo, J. Gao and S. D. Peyerimhoff, J. Chem. Phys., 2000, 112, 5530.

26 P. Su and H. Li, J. Chem. Phys., 2009, 131, 014102.

27 P. Fowler and A. Sadlej, Mol. Phys., 1992, 77, 709-725.

28 D. M. Bishop and M. Dupuis, Mol. Phys., 1996, 88, 887-898.

29 T. G. A. Heijmen, R. Moszynski, P. E. S. Wormer and A. van der Avoird, Mol. Phys., 1996, 89, 81-110.

30 B. Skwara, A. Kaczmarek, R. W. Góra and W. Bartkowiak, Chem. Phys. Lett., 2008, 461, 203-206.

31 R. W. Góra, R. Zaleśny, A. Zawada, W. Bartkowiak, B. Skwara, M. G. Papadopoulos and D. L. Silva, J. Phys. Chem. A, 2011, 115, 4691-4700.

32 R. W. Góra and B. Błasiak, J. Phys. Chem. A, 2013, 117, 6859-6866.

33 A. Baranowska-Łaczzkowska, B. Fernandez and R. Zaleśny, J. Comput. Chem., 2013, 34, 275-283.

34 M. Medveď, Š. Budzak, A. D. Laurent and D. Jacquemin, J. Phys. Chem. A, 2015, 119, 3112-3124.

35 B. Champagne and B. Kirtman, in Theoretical approach to the design of organic molecular and polymeric nonlinear optical materials, ed. H. Singh, Academic Press, 2001, pp. 63-126.

36 R. Zaleśny, M. Garcia-Borrás, R. Góra, M. Medved and J. M. Luis, Phys. Chem. Chem. Phys., 2016, 18, 22467-22477.

37 R. Zaleśny, M. Medved, R. W. Góra, H. Reis and J. M. Luis, Phys. Chem. Chem. Phys., 2018, 20, 19841-19849.

38 G. Cavallo, P. Metrangolo, R. Milani, T. Pilati, A. Priimagi, G. Resnati and G. Terraneo, Chem. Rev., 2016, 116, 2478-2601.

39 T. M. Beale, M. G. Chudzinski, M. G. Sarwar and M. S. Taylor, Chem. Soc. Rev., 2013, 42, 1667-1680.

40 L. P. Wolters, P. Schyman, M. J. Pavan, W. L. Jorgensen, F. M. Bickelhaupt and S. Kozuch, Wiley Interdiscip. Rev.: Comput. Mol. Sci., 2014, 4, 523-540.

41 P. Politzer, J. S. Murray and T. Clark, in $\sigma$-Hole Bonding: A Physical Interpretation, ed. P. Metrangolo and G. E. Resnati, Springer International Publishing, 2015, pp. 19-42.

42 M. H. Kolář and P. Hobza, Chem. Rev., 2016, 116, 5155-5187.

43 H. Wang, W. Wang and W. J. Jin, Chem. Rev., 2016, 116, 5072-5104.

44 P. S. Ho, in Biomolecular Halogen Bonds, ed. P. Metrangolo and G. E. Resnati, Springer International Publishing, 2015, pp. 241-276.

45 A. Abate, M. Saliba, D. J. Hollman, S. D. Stranks, K. Wojciechowski, R. Avolio, G. Grancini, A. Petrozza and H. J. Snaith, Nano Lett., 2014, 14, 3247-3254.
46 O. Dumele, N. Trapp and F. Diederich, Angew. Chem., Int. Ed., 2015, 54, 12339-12344.

47 W. Zierkiewicz, R. Wieczorek, P. Hobza and D. Michalska, Phys. Chem. Chem. Phys., 2011, 13, 5105-5113.

48 G. Berger, J. Soubhye and F. Meyer, Polym. Chem., 2015, 6, 3559-3580.

49 M. Saccone, G. Cavallo, P. Metrangolo, G. Resnati and A. Priimagi, in Halogen-Bonded Photoresponsive Materials, ed. P. Metrangolo and G. E. Resnati, Springer International Publishing, 2015, pp. 147-166.

50 S. Schindler and S. M. Huber, in Halogen Bonds in Organic Synthesis and Organocatalysis, ed. P. Metrangolo and G. E. Resnati, Springer International Publishing, 2015, pp. 167-203.

51 Y. Lu, Y. Wang and W. Zhu, Phys. Chem. Chem. Phys., 2010, 12, 4543-4551.

52 T. Clark, M. Hennemann, J. S. Murray and P. Politzer, J. Mol. Model., 2007, 13, 291-296.

53 P. Politzer, J. S. Murray and P. Lane, Int. J. Quantum Chem., 2007, 107, 3046-3052.

54 J. S. Murray, P. Lane and P. Politzer, J. Mol. Model., 2009, 15, 723-729.

55 V. Angarov and S. Kozuch, New J. Chem., 2018, 42, 1413-1422.

56 M. Hou, Q. Li and S. Scheiner, ChemPhysChem, 2019, 20, 1978-1984.

57 L. P. Wolters and F. M. Bickelhaupt, ChemistryOpen, 2012, 1, 96-105.

58 M. E. Wolf, B. Zhang, J. M. Turney and H. F. Schaefer, Phys. Chem. Chem. Phys., 2019, 21, 6160-6170.

59 P. M. J. Szell, G. Cavallo, G. Terraneo, P. Metrangolo, B. Gabidullin and D. L. Bryce, Chem. - Eur. J., 2018, 24, 11364-11376.

60 P. Hobza and Z. Havlas, Chem. Rev., 2000, 100, 4253-4264.

61 J. Joseph and E. D. Jemmis, J. Am. Chem. Soc., 2007, 129, 4620-4632.

62 J. Joy, E. D. Jemmis and K. Vidya, Faraday Discuss., 2015, 177, 33-50.

63 V. Oliveira, E. Kraka and D. Cremer, Inorg. Chem., 2017, 56, 488-502.

64 W. Wang and P. Hobza, J. Phys. Chem. A, 2008, 112, 4114-4119.

65 S. Kozuch and J. M. L. Martin, J. Chem. Theory Comput., 2013, 9, 1918-1931.

66 D. M. Bishop, M. Hasan and B. Kirtman, J. Chem. Phys., 1995, 103, 4157-4159.

67 B. Kirtman, J. M. Luis and D. M. Bishop, J. Chem. Phys., 1998, 108, 10008-10012.

68 M. Gutowski, F. B. van Duijneveldt, G. Chałasiński and L. Piela, Mol. Phys., 1987, 61, 233-247.

69 W. A. Sokalski, S. Roszak and K. Pecul, Chem. Phys. Lett., 1988, 153, 153-159.

70 S. M. Cybulski, G. Chałasiński and R. Moszyński, J. Chem. Phys., 1990, 92, 4357-4363.

71 G. Chałasiński and M. M. Szcześniak, Chem. Rev., 1994, 94, 1723-1765.

72 D. Bishop, Adv. Chem. Phys., 1998, 104, 1-40.

73 J. M. L. J. Martí, M. Duran, J. L. Andrés and B. Kirtman, J. Chem. Phys., 1998, 108, 4123-4130. 
74 D. M. Bishop, M. Hasan and B. Kirtman, J. Chem. Phys., 1995, 103, 4157.

75 C. Eckart, Phys. Rev., 1926, 28, 711.

76 J. M. Luis, M. Duran, J. L. Andrés, B. Champagne and B. Kirtman, J. Chem. Phys., 1999, 111, 875.

77 M. Gutowski, F. V. Duijneveldt, G. Chałasiński and L. Piela, Mol. Phys., 1987, 61, 233-247.

78 W. A. Sokalski, S. Roszak and K. Pecul, Chem. Phys. Lett., 1988, 153, 153-159.

79 S. M. Cybulski, G. Chałasiński and R. Moszyński, J. Chem. Phys., 1990, 92, 4357-4363.

80 G. Chałasiński and M. M. Szcześniak, Mol. Phys., 1988, 63, 205-224.

81 F. B. van Duijneveldt, J. G. C. M. van Duijneveldt-van de Rijdt and J. H. van Lenthe, Chem. Rev., 1994, 94, 1873-1885.

82 R. Moszyński, S. Rybak, S. Cybulski and G. Chałasiński, Chem. Phys. Lett., 1990, 166, 609-614.

83 R. A. Kendall, T. H. Dunning and R. J. Harrison, J. Chem. Phys., 1992, 96, 6796-6806.

84 K. A. Peterson, D. Figgen, E. Goll, H. Stoll and M. Dolg, J. Chem. Phys., 2003, 119, 11113-11123.

85 M. J. Frisch, G. W. Trucks, H. B. Schlegel, G. E. Scuseria, M. A. Robb, J. R. Cheeseman, G. Scalmani, V. Barone, B. Mennucci, G. A. Petersson, H. Nakatsuji, M. Caricato, X. Li, H. P. Hratchian, A. F. Izmaylov, J. Bloino, G. Zheng, J. L. Sonnenberg, M. Hada, M. Ehara, K. Toyota, R. Fukuda, J. Hasegawa, M. Ishida, T. Nakajima, Y. Honda, O. Kitao, H. Nakai, T. Vreven, J. A. Montgomery, Jr., J. E. Peralta,
F. Ogliaro, M. Bearpark, J. J. Heyd, E. Brothers, K. N. Kudin, V. N. Staroverov, R. Kobayashi, J. Normand, K. Raghavachari, A. Rendell, J. C. Burant, S. S. Iyengar, J. Tomasi, M. Cossi, N. Rega, J. M. Millam, M. Klene, J. E. Knox, J. B. Cross, V. Bakken, C. Adamo, J. Jaramillo, R. Gomperts, R. E. Stratmann, O. Yazyev, A. J. Austin, R. Cammi, C. Pomelli, J. W. Ochterski, R. L. Martin, K. Morokuma, V. G. Zakrzewski, G. A. Voth, P. Salvador, J. J. Dannenberg, S. Dapprich, A. D. Daniels, O. Farkas, J. B. Foresman, J. V. Ortiz, J. Cioslowski and D. J. Fox, Gaussian 09, Revision D.01, Gaussian Inc., Wallingford, CT, 2009.

86 J. M. Luis, M. Duran, J. L. Andrés, B. Champagne and B. Kirtman, J. Chem. Phys., 1999, 111, 875-884.

87 J. M. Luis, M. Duran, B. Champagne and B. Kirtman, J. Chem. Phys., 2000, 113, 5203-5213.

88 M. W. Schmidt, K. K. Baldridge, J. A. Boatz, S. T. Elbert, M. S. Gordon, J. H. Jensen, S. Koseki, N. Matsunaga, K. A. Nguyen, S. Su, T. L. Windus, M. Dupuis and J. A. Montgomery, J. Comput. Chem., 1993, 14, 1347-1363.

89 R. W. Gora, EDS package, Revision 2.8.3, Wrocław, Poland, 1998-2008.

90 R. Zaleśny, A. Baranowska-Łączkowska, M. Medved and J. M. Luis, J. Chem. Theory Comput., 2015, 11, 4119-4128.

91 P. Hobza and K. Muller-Dethlefs, Non-Covalent Interactions, The Royal Society of Chemistry, 2009, pp. P001-P226.

92 S. Grimme, J. Chem. Phys., 2003, 118, 9095-9102.

93 K. E. Riley and P. Hobza, J. Chem. Theory Comput., 2008, 4, 232-242. 